\title{
Corela
}

Cognition, représentation, langage

HS-1 1 | 2012

RJC Cotexte, contexte, situation

\section{Le sous-titrage de film ou la prise en compte d'une dialectique contextuelle}

Eve Vayssière

\section{OpenEdition}

\section{Journals}

Édition électronique

URL : http://journals.openedition.org/corela/2100

DOI : $10.4000 /$ corela. 2100

ISSN : 1638-573X

\section{Éditeur}

Cercle linguistique du Centre et de I'Ouest - CerLICO

Référence électronique

Eve Vayssière, «Le sous-titrage de film ou la prise en compte d'une dialectique contextuelle », Corela [En ligne], HS-11 | 2012, mis en ligne le 20 avril 2012, consulté le 20 avril 2019. URL : http:// journals.openedition.org/corela/2100 ; DOI : 10.4000/corela.2100

Ce document a été généré automatiquement le 20 avril 2019

\section{(c) (i) (2)(2)}

Corela - cognition, représentation, langage est mis à disposition selon les termes de la licence Creative Commons Attribution - Pas d'Utilisation Commerciale - Partage dans les Mêmes Conditions 4.0 International. 


\title{
Le sous-titrage de film ou la prise en compte d'une dialectique contextuelle
}

\author{
Eve Vayssière
}

\section{Introduction}

1 Le sous-titrage de film est une traduction analysée en général selon deux approches. Une première approche est celle de la comparaison sous-titrage / doublage, telle que par exemple celle de Teresa Tomaszkiewicz ${ }^{1}$. Une seconde approche met en avant les contraintes techniques, à l'instar de Daniel Becquemont ${ }^{2}$ dans un article où ces dernières sont tenues pour responsables de la mauvaise qualité de la traduction sous-titrante qu'elles empêcheraient d'être fidèle à l'original.

2 J'analyse exclusivement le sous-titrage de film et ne m'intéresse aux contraintes techniques dont Becquemont fait de manière exhaustive l'inventaire que par le biais de leur dépassement.

3 Le sous-titrage de film peut se définir comme une des manières de rendre accessible le sens et le contenu d'un film à un public qui ne maîtrise pas la langue dans laquelle se déroulent les échanges des personnages ou dans laquelle est formulé le commentaire (d'un documentaire, par exemple). C'est une traduction qui passe de l'oral inintelligible pour le spectateur cible à l'écrit intelligible et se fait ainsi transcription.

4 Nous pourrons constater que les notions d'interactions, de contexte situationnel et extralinguistique sont fondamentales pour l'analyse du sous-titrage de film. Mon approche correspond à ce que les organisateurs ont intitulé l'axe trois :

«Dans une perspective pragmatico-énonciative et interactionnelle, les communications pourront porter sur la notion de situation dans laquelle un énoncé, un dialogue, un discours, etc. - qu'il soit écrit ou oral - se situe afin de poser des hypothèses sur l'importance du contexte situationnel ou extralinguistique, sur ses motifs et ses effets (circonstances d'un acte d'énonciation, identité des interlocuteurs, intention des locuteurs, etc.). Ce troisième axe inclut également 
toutes les études qui ont pour objets les indices pertinents pour les conditions de

compréhension ou d'interprétation en situation. » l'élaboration du sous-titrage de film.

\section{Le contexte intrafilmique}

\subsection{Unités de sens}

9 Si l'on dit que le sous-titrage de film est une traduction lacunaire, c'est qu'il y erreur sur l'original. On oublie trop souvent qu'il s'agit là d'une traduction audiovisuelle et non littéraire. C'est à dire que le traducteur ne va pas s'appuyer uniquement sur les dialogues mais sur tout ce qui fait sens dans le film.

10 J'appellerai cela le langage cinématographique. En effet, les dialogues, les images, le son, les différents niveaux présents au sein du contexte intrafilmique sont porteurs de sens. Si l'on considère le film, on peut se rendre compte qu'il est une sorte de poupée russe, composé d'unités toujours plus petites qui pourraient faire sens à elles seules mais dont l'imbrication est porteuse du sens souverain, celui du film dans sa globalité. Le film pourrait être défini comme le montage de séquences comprenant elles-mêmes des scènes composées d'images, de son et d'unités de sens proprement linguistiques. Les scènes sont ce que j'appelle les unités phénoménologiques globales: on ne comprend pas chaque élément en tant que tel mais leur articulation, c'est cette articulation qui est perçue par le spectateur et qui lui permet d'accéder au sens.

11 Le sous-titre est une nouvelle unité au sein du film : la traduction d'unités globales par des unités de sens langagières. Il calque la prosodie, en apparaissant et disparaissant au même rythme que les unités linguistiques, tout en respectant les unités cinématographiques et l'enchaînement des plans. Pour cela, le traducteur s'appuie sur un savoir partagé et nous assistons à une sorte d'ajustement entre traducteur et spectateurs. Cet ajustement est à l'oeuvre lorsque le traducteur décide d'impliciter ou d'expliciter certaines informations contenues dans les propos des personnages. Si ces informations sont également accessibles par l'image, elles ne seront en général pas traduites. D'une part du fait des contraintes techniques de temps d'apparition du sous-titre à l'écran, d'autre part afin d'éviter une redondance entre image et sous-titre qui n'était pas perçue 
à l'oral. Cela rendrait le sous-titre vain. Dans l'exemple suivant, tiré du film La vie des autres, nous pourrons voir comment le sous-titre s'appuie sur l'image.

[Georg vient de fêter son anniversaire et déballe ses cadeaux]

Christa-Maria : Tes amis n'ont pas beaucoup de goût...

Georg: Tu es vraiment injuste. Regarde ce beau gratte-dos.

- C'est une fourchette à salade!

- N'empêche, c'est magnifique ! Regarde-moi ça ! J'écrirai ma prochaine pièce avec. ${ }^{4}$

12 Si l'on considère cette liste de sous-titres, Georg envisage d'écrire sa prochaine pièce avec une fourchette à salade. Or, si l'on regarde l'extrait du film concerné, on se rend compte qu'il s'empare au même moment d'un stylo. Nous pouvons ainsi constater que sans les images, cette liste de sous-titres est incompréhensible. Dans une situation de conversation, locuteur et destinataire partagent le même référentiel ${ }^{5}$ et n'ont donc pas besoin de situer ou contextualiser la situation. Il en va de même pour les personnages mis en scène : Georg sait que Christa-Maria voit les objets qu'il manipule. Les sous-titres sont une traduction «en contexte » et de ce fait perdent leur intelligibilité sans le support filmique. Cet exemple nous permet également de percevoir une relation d'interdépendance dans laquelle le sous-titre s'appuie tout autant sur le contexte qu'il en dépend. Sans l'image, le sous-titre ne fait plus sens, sans le sous-titre, les spectateurscible ne peuvent plus suivre le dialogue. Le sous-titre n'est plus seulement une incrustation dans l'image mais partie intégrante du film pour tout spectateur-cible. En cela, on peut parler d'un rapport dynamique du sous-titre au contexte filmique.

13 La notion de contexte intrafilmique fait référence à la prise en compte du langage cinématographique comme un tout permettant l'accès au sens. Comme l'écrit Robert Bresson dans Notes sur le cinématographe $e^{6}$, un film de cinéma n'est pas une pièce de théâtre filmée. Sous cette apparente évidence se cache une vérité fondamentale. On oublie trop souvent que dans un film, il y a des acteurs, des images, du son mais que tout cela a été articulé par le montage. Le montage est le squelette du film, il en détermine les enchaînements dans un but précis. Le sous-titrage n'est pas une traduction des paroles au fur et à mesure qu'elles s'égrènent mais la traduction d'une oeuvre intégrale, c'est la raison pour laquelle le traducteur va parfois expliciter des scènes au regard de celles dans lesquelles elles s'insèrent. A travers ces notes, Bresson nous donne sa vision de praticien et écrit sans l'étiqueter, l'importance des interactions au sein d'un film :

"Il faut qu'une image se transforme au contact d'autres images comme une couleur au contact d'autres couleurs. Un bleu n'est pas le même bleu à côté d'un vert, d'un jaune, d'un rouge. $»^{7}$

14 La transformation est ici due à la mise en parallèle de différentes couleurs parce que cette disposition les fera percevoir différemment. Or, la langue fait partie du film et à ce titre, elle ne peut pas être isolée, sans quoi le sens se perd, de la même façon que le bleu sera perçu différemment en fonction de l'environnement dans lequel il s'insère. Bresson poursuit :

«Film de cinématographe où les images, comme les mots du dictionnaire, n'ont de pouvoir et de valeur que par leurs position et relation. $»^{8}$

Nous retrouvons ici la notion d'interaction et celle de contexte. Le sous-titre est dynamique parce qu'il rentre dans ce jeux d'interactions et se fait vecteur du sens pour le spectateur cible. 


\subsection{Compensation des contraintes}

16 Nous avons vu la relation d'interdépendance entretenue par le film et sa traduction. Le support audiovisuel sur lequel s'appuie le sous-titre est aussi source de contraintes techniques. Le contexte va à nouveau jouer un rôle fondamental. Il ne va pas être seulement nécessaire à la compréhension du film mais va permettre au traducteur de dépasser les contraintes techniques, lui permettant l'implicitation d'informations d'ores et déjà comprises par le spectateur grâce au contexte dans lequel s'insèrent les répliques.

17 Les contraintes techniques sont: de temps d'apparition du sous-titre à l'écran, de synchronisation propos/apparition et disparition du sous-titre, de non-chevauchement de plusieurs plans par le sous-titre, ainsi que de la taille du sous-titre qui ne peut excéder deux lignes. Nous aborderons deux procédés particulièrement instructifs: la condensation/implicitation et l'explicitation.

\subsubsection{La condensation/implicitation}

18 La condensation/implicitation est une sorte de résumé à propos duquel Jean-Paul Bernié écrit qu'il passe par la paraphrase et la hiérarchisation des informations. Par résumé, nous n'entendons pas celui de tout un dialogue mais la condensation d'un propos. Un ancien sous-titrage du film de Harun Farocki ${ }^{10}$ portant sur la chute de Ceauşescu est révélateur de ce point de vue: la personne chargée de sa traduction n'a pas traduit les dialogues. Après $10 \mathrm{mn}$ de visionnage apparaît soudain un sous-titre «Ils ne sont pas contents ». Information déjà connue de toute personne voyant des manifestations et des personnes qui pleurent. Il ne s'agissait pas d'un résumé mais d'un constat sur les images et les propos tenus. La condensation réalisée par le traducteur ne donnera pas lieu à une d'interprétation de la scène mais contiendra les informations contenues dans les propos des personnages nécessaires à la compréhension du film. Il n'y aura pas suppression mais bien condensation comme dans l'exemple suivant :

Das habe ich doch schon zur Protokoll gegeben.

[Mais je l'ai déjà donné au protocole.]

=> C'est dans ma déposition.

Dans cet exemple tiré du premier chapitre ${ }^{11} \mathrm{du}$ film, on assiste à un interrogatoire. L'exemple choisi est la réponse que le prévenu fait à Wiesler, agent de la Stasi, après que celui-ci lui a demandé ce qu'il faisait le jour où un de ses amis s'est enfui de R.D.A. Le propos tenu et sa traduction font appel au réseau de connaissances ou référentiel commun des spectateurs, ce qui rend toute explicitation fortuite. Le propos original du détenu est qu'il a déjà fait l'action consistant à donner son emploi du temps alors que la traduction conserve uniquement le résultat de cette action : son emploi du temps est dans sa déposition. Un résultat est précédé d'une action, ce savoir permet au traducteur de condenser le message, de faire passer dans l'implicite ce qui était explicite dans l'original et de faire ainsi un sous-titre court en se servant du référentiel des spectateurs.

\subsubsection{L'explicitation}

Inversement, le traducteur est parfois amené à expliciter le contenu du message. Dans ce cas, le traducteur insère l'information induite par l'image dans sa traduction. A ce propos, D. Becquemont écrit que : 
«Cette nécessité de saisir dans l'acte d'adaptation, non pas l'énoncé verbal isolé, mais l'ensemble des interactions entre visuel et verbal, est l'une des conditions fondamentales pour effectuer un bon sous-titrage. $»^{12}$

C'est de cette interaction entre «visuel et verbal» ou entre image et langue que vont naître les profits apportés par la traduction. Le traducteur sera en effet amené à traduire des éléments de l'image mais aussi les éléments du contexte comme dans un extrait du chapitre $9 \mathrm{du}$ film, où Wiesler, agent de la Stasi qui espionne Georg et Christa-Maria, a l'intention de donner un rapport à Grubitz concernant Georg Dreymann. Avant qu'il puisse parler, Grubitz lui parle d'une thèse qui vient de lui être remise décrivant les traitements les plus efficaces administrés aux artistes «subversifs". Il lui décrit ce qui serait fait à Georg et dans quel état il sortirait de détention. A ce moment-là, on voit le visage de Wiesler changer et un plan passe du dossier qu'il tient sous la table contenant de quoi faire incarcérer Georg jusqu'à son visage. C'est à ce moment qu'il décide de taire la vérité et quelques dizaines de secondes plus tard Grubitz lui dit :

Grubitz : Irgend etwas sagst du mir nicht.

[Il y a quelque chose que tu ne me dis pas]

$\Rightarrow>$ Tu me caches quelque chose.

La traduction choisie est d'une part plus idiomatique que la traduction littérale et fait d'autre part référence au comportement de Wiesler. Ce sont deux extraits d'une séquence assez longue. Cette longueur implique une déperdition d'informations compensée ici par la traduction plus fidèle à la séquence qu'à la réplique qui faisait l'objet de la traduction. Le plan qui passe du dossier au visage de Wiesler nous fait comprendre qu'il va le cacher. Ainsi, la traduction correspond extrêmement bien, non seulement au contexte, mais aussi à l'attente des spectateurs. On pourrait même aller plus loin, et dire que le choix du verbe cacher correspond à tout le film. Que fait Wiesler, agent de la Stasi, sinon chercher à découvrir ce que les gens cachent en les espionnant.

\section{Le contexte extrafilmique}

3 Nous avons vu le rapport d'interdépendance entre sous-titre et film par le biais du contexte intrafilmique. Le contexte extrafilmique a également son importance: c'est parce que la technologie a avancé que les films sont devenus parlants et c'est parce qu'ils sont devenus parlants qu'il n'a plus été possible de les exporter comme auparavant (toute proportion gardée du fait des différences culturelles entre pays) et qu'il a donc fallu trouver des moyens de traduire les propos des personnages. de l'avènement du film parlant sur les procédés narratifs mais également l'Histoire l'entre-deux-guerres, la seconde guerre mondiale, la France parmi les vainqueurs, les deux Allemagnes. Le genre de film, le réalisateur et le budget ont également leur place dans ce contexte extrafilmique.

Ces époques sont marquées par des mouvements cinématographiques qui en sont souvent le reflet. A savoir par exemple l'expressionisme, les films de propagande, ceux d'aprèsguerre. Le traducteur ne fera pas le même travail qu'il s'agisse d'un film des décombres d'Allemagne de l'Est, tel que Die Mörder sind unter uns de Wolfgang Staudte, sorti en 1946, ou d'un film d'Allemagne de l'Ouest à la même époque, film campagnard de divertissement louant la loyauté du paysan et le retour à des valeurs conservatrices et familiales. A la même époque apparaît en France La Nouvelle Vague dont Raymond Borde 
${ }^{13}$ dit qu'elle a été une crise du langage. Les registres de ces films diffèrent et leur traduction également. Avec la Nouvelle Vague, on a assisté à l'éclatement du découpage, l'apparition de parenthèses, le montage court. Autant de modifications et de caractéristiques dont va tenir compte le traducteur et auxquelles il va être confronté. Eclatement du langage cinématographique mais aussi de la langue. Bresson refusait de travailler avec de vrais acteurs, et leur imposait une diction dont toute intonation était absente. Quant au montage court dont parle R. Borde, il implique une difficulté de taille pour le sous-titreur qui doit respecter dans le positionnement des sous-titres, tout changement de plan.

Le contexte extrafilmique veut également qu'à chaque "remasterisation" d'un film ancien, le sous-titrage, tel un produit périssable, soit refait. Une action qui pourait appraitre positive si elle ne s'accompagnait pas de la destruction des versions sous-titrées précédentes. Disparition qui rend impossible la comparaison entre deux versions. Comment savoir ce qui a été interprété différemment, ce qui a été «remis au goût du jour » ? Car c'est bien de cela dont il s'agit. Le contexte extrafilmique change et implique ces re-traductions multiples. Les références et le vocabulaire du public évoluent, notamment en ce qui concerne l'argot. Le film sur lequel je me suis appuyée est trop récent pour qu'il y ait eu plusieurs versions de sous-titrage. Néanmoins, certaines expressions employées sont déjà plus ou moins désuètes, comme dans cet exemple :

Dreymann : Ich glaube, er hat einen Narren an dir gefressen.

[Je crois qu'il a mangé un fou sur toi]

$\Rightarrow>$ Je crois qu'il a le béguin pour toi.

Ces deux métonymies font référence à un épisode amoureux et ont en commun de désigner quelqu'un comme étant fortement attiré par une autre personne. Dans l'expression allemande comme dans la française, la personne n'est pas lucide. En allemand, la personne a avalé un fou. En français, qu'il s'agisse de la coiffe de femmes qui se vouaient à Dieu ou de la coiffe que l'on faisait porter aux enfants sous leur bonnet, et qui avait apparemment tendance à glisser cachant ainsi leurs yeux, il est question de se laisser convaincre à tort ou de ne plus y voir clair. Certains adolescents ne connaissent plus la signification de l'expression française et il ne serait pas surprenant d'observer une modification de ce sous-titre dans quelques années.

\section{Conclusion}

28 Nous avons vu que les images comme les informations contextuelles permettent au traducteur sous-titreur de sélectionner, parmi les informations langagières, celles qu'il va traduire. C'est à dire celles que les images, le contexte ou encore le savoir partagé d'une communauté de personnes ne suffisent pas à comprendre. Cette caractéristique rend le sous-titrage de film possible tout comme elle le rend dépendant de l'époque dans laquelle il a été élaboré.

29 J'ai montré de quelle façon le traducteur tenait compte du contexte dans lequel s'insérait l'acte de parole, de l'interaction entre les divers éléments du langage cinématographique et des éléments de compréhension dores et déjà présents dans l'image et de quelle façon cela induisait un rapport dynamique entre le sous-titrage et les contextes intra- et extrafilmiques. 


\section{BIBLIOGRAPHIE}

BECQUEMONT Daniel (1996), "Les Sous-titrages cinématographiques : contraintes, sens, servitudes", pp. 145-155 in Les transferts linguistiques dans les médias audiovisuels, Paris (Presses universitaires du Septentrion)

BERNIE Jean-Paul (1993), Raisonner pour résumer. Une approche systémique du texte, Berne (Peter Lang S.A.)

BORDE Raymond, préface de COURTADE François (1978), Les malédictions du cinéma français, Paris (Alain Moreau) pp. 7-21

BRESSON Robert (1995), Notes sur le cinématographe, Paris (Gallimard)

MOUNIN Georges (2006), Dictionnaire de la linguistique, Mercuès, (Quadrige - PUF) 11974

TOMASZKIEWICZ Teresa (1993), Les opérations linguistiques qui sous-tendent le processus de soustitrage des films, Poznan, (Wydawnictwo Naukowe Uniwersytetu Im. Adama Mickiewicza W Poznaniu)

FILMOGRAPHIE

FAROCKI Harun, UJICA Andrei (1992), Videogramme einer Revolution, Allemagne

HENCKEL VON DONNERSMARCK Florian (2007), Das Leben der anderen, Allemagne (Ocean Film)

\section{NOTES}

1. TOMASZKIEWICZ (1993)

2. BECQUEMONT (1996)

3. HENCKEL VON DONNERSMARCK (2007)

4. HENCKEL VON DONNERSMARCK (2007)

5. "La langue, en elle-même ensemble structuré de formes, n'a de raison d'être que dans la mesure où elle est en relation avec l'expérience que les locuteurs ont du monde." MOUNIN (2006)

6. BRESSON (1995)

7. BRESSON (1995) p. 22

8. BRESSON (1995) p. 22

9. BERNIE (1993)

10. FAROCKI, UJICA (1992)

11. Un film est composé de séquences appelées « chapitres ».

12. BECQUEMONT (1996) p. 152

13. BORDE (1978) 


\section{RÉSUMÉS}

La traduction sous-titrante est soumise à une dialectique contextuelle. Le traducteur tient en effet compte de deux types de contextes : un intra- et un extrafilmique. La notion de contexte intrafilmique fait référence à la prise en compte du langage cinématographique (images, langue, bande-son, situations, etc.) dont les éléments participent à la formation d'unités phénoménologiques globales par lesquelles le spectateur accède au sens du film. Le contexte extrafilmique désigne l'entour $\mathrm{du}$ film, à savoir notamment l'Histoire, les pratiques traductologiques, les techniques cinématographiques, la culture du spectateur-cible. Le caractère éphémère de ce type de traduction est la conséquence du rapport dynamique entretenu par le sous-titrage et le contexte extrafilmique dans lequel il s'insère.

Subtitle translation is subjected to a dialectics of contexts. Indeed, the translator must take both intra-filmic and extra-filmic contexts into account. The notion of intra-filmic context implies taking into account the cinematographic language (images, language, sound), whose elements generate whole phenomenological units which give the spectator access to the meaning of the film. The extra-filmic context, on the other hand, refers to what surrounds the film, such as history, trends and techniques in film, or the culture of the target audience. The specifically transient aspect of this kind of translation results from the dynamic relationship between subtitling and the extra-filmic context within which it lies.

\section{INDEX}

Mots-clés : contexte, traduction, unité de sens, phénoménologie, sous-titre, langage cinématographique

Keywords : context, translation, meaning, subtitle, dialectic, interaction, cinematographic language, image

\section{AUTEUR}

\section{EVE VAYSSIÈRE}

Université Paris 3 - Sorbonne Nouvelle 